\title{
Influence Factors and Construction Strategy of R \& D Team Psychological Safety
}

\author{
Xiang-Dong Feng ${ }^{1}$, Bao-Liang Cao ${ }^{1}$, Nian-Bai Li ${ }^{1}$, Han-Song Chen², Wei $\mathrm{Yu}^{2}$,a, \\ ${ }^{1}$ Shandong Weifang Tobacco Company, P R China \\ ${ }^{2}$ Department of Business, Shandong University of Finance and Economics, P R China \\ alongkouyuwei@sina.com \\ ${ }^{*}$ Corresponding author
}

Keywords: R \& D team, Psychological safety, Human management.

\begin{abstract}
Psychological safety is one of the important variables in determining the overall performance of the team. Influence factors on R \& D team psychological safety include social culture, team management, learning competition, personnel personality and the characteristics of research and development work. Therefore, construction of psychological safety should based on team culture, humane management, team learning competition, introduction of psychological security education and the implementation of inter organizational cooperation.
\end{abstract}

\section{Introduction}

Fast and effective communication in R \& D team is the key to increase enterprise's knowledge and enhance its market potential. In the previous studies, internal knowledge, scientific research personnel communication skills, learning ability and enterprise hardware facilities all can affect R \& D team communication effect. Although there are numerous research achievements, the psychological factors are not enough. The psychological factors of team members can affect team communication in subtle ways, among them, team psychological safety is a key factor. Team psychological safety refers to the members of the team belief, which allows members to get rid of the interpersonal relationship risk concerns to express the views and opinions. For example, when point out colleagues' error, he don't worry about the injury of relations between each other. When put forward in the new ideas, he could not be targets for public ridicule because it is not feasible. Team psychological safety provides a new perspective and tool for the development team of enterprise soft environment.

\section{Influence Factors of R \& D Team Psychological Safety}

Many factors can influence R \& D team psychological, including the characteristics of social culture and sub culture within the organization, organization atmosphere, management, scientific research personnel and characteristics of the knowledge of science and technology, these factors all determine the strength of team psychological safety.

\section{Traditional Culture}

As a specific element in the economic society, the internal activities of enterprises cannot do without the influence of traditional culture. Some of the elements of traditional culture have a negative impact on the psychological safety of $\mathrm{R} \& \mathrm{D}$ personnel.

First, the demands of "harmony" and value of "MianZi" has significant influence on the communication within the team. For example, high-tech research and development is often conducted by the method of "trial and error". To point out error means denial of early input of team and R \& D partners, which can hurt others' face and cause internal disharmony, and then make team member have to worry about his opinion. Second, culture of "circle" can enlarge interpersonal risk. The existence of "circle" is the internal common phenomenon within organization. The "circle" has highly consistent interests and external sense of exclusion. Once one of them has be corrected, it can easily lead to the whole hostility from the "circle". The magnifying effect greatly increases the potential risk of interpersonal relationship 
for critics. Third, the concerns of "early bird" inhibit e team members to express their views freely. When they find error signs without directly related to their own interests, most people will choose others to act as "early bird" instead of their own act as "focus".

\section{Team Management and Leadership Style}

Internal leadership team and the management style have a direct impact on the psychological sense of security. According to the leader member exchange theory, the performance of team members are closely related management and internal services. Transactional leadership has character of contingent reward and exception management, under this leadership style, team members tend to complete the job in order to get a prior agreement of the reward instead of put forward the views and suggestions by relationship risk. Transformational leadership has character of charismatic leadership, vision incentives, intellectual stimulation and individual consideration, organization atmosphere more harmonious and relaxed, mental resources of members can be fully mobilized for the completion of the goals of the organization, team members are not only willing put forward their own views for specific problems, these suggestions will always be smooth adoption.

The improper disposal of internal management in R \& D team members will increase the perceived injustice in the team and increase their mutual awareness and sense of distrust. Management method of favor one more than another will lead to interpersonal relationship are complicated and opportunism behavior. In addition, many managers in reality tend to use a variety of ways to understand the idea of the members by acquiescence and even encourage snitch and so on, which will greatly reduce the team psychological safety, make the communication within the organization is limited within a given small circle, thereby affect the exchange effect.

\section{Learning Competition in The Team}

As the intelligence and knowledge intensive organization, R \& D team must keep the absorption and application of new knowledge. In the process of the acceptance of new knowledge, learning competition can hardly be avoided because of individuals' different acceptance ability. Team learning competition has "double-edged" effect. On the one hand, learning competition can greatly mobilize members' enthusiasm and form "innovation friendly" atmosphere, increase the team's knowledge and flexible capacity, on the other hand, it can strengthen the consciousness of competition in organizations and hinder the flow of knowledge within the team, and lead to duplication of work and ineffective phenomenon. Especially when the internal evaluation and incentive take group as the basic unit, closed tendency caused by learning competition will be more obvious. In this case, when the researchers put forward advice to other groups, he will take much interpersonal relationship risk, including non-cooperative behaviors from information receiver and dissatisfaction from members of own group because of information leakage.

\section{The Personality Characteristics of R \& D Personnel}

R \& D personnel with strong creativity are well educated and have a significant personality traits, such as higher self-efficacy and toughness. Combined with pursuit of quality and detail in the research and development work, they are easy to develop strong self-esteem and the pursuit of the perfect personality, which can break through the technical difficulties effectively and deal with unexpected events. But it also can reject various sounds. When other team members are expected his advice has no effect but need to take the risk of interpersonal relationship, his psychological security will reduce.

\section{The Characteristics of Knowledge and R \& D Work}

Knowledge of science and technology update quickly, at the same time, research and development work has characteristics of fuzzy front, uncertainty and high failure rate, which make the researchers are faced with enormous psychological pressure. The psychology research indicates that, the psychological pressure of the individual is very easy to produce negative response to external things and even lead to radical reaction, which will increase the probability of deterioration of group interpersonal relationship. At the same time, the success of product development needs a series of supporting and promoting mechanism within the organization. According to the principle of matching of power and knowledge, R \& D personnel should have the power to ensure the work, which includes a specific range of control over 
resources, emergency disposal right in research and development process. If the required power loss, $\mathrm{R} \&$ D personnel psychological sense of security also decreased.

\section{Construction Strategy of the R \& D Team Psychological Safety}

\section{Shape "Sub Culture" Within the Team to Encourage Knowledge Sharing Behavior}

As the norms of behavior and values within the team, team culture has a significant effect of regulating and guiding role, even avoid some negative social culture in the very great degree. The development of high and new technologies and products more and more need researchers to take "research team" to solve the technical problem, and only adequate communication can ensure team learning and knowledge sharing. Innovation-friendly team culture includes open cooperation, mutual trust, knowledge sharing and team learning factors. Open cooperation means to break the inherent mode of mind and accept different sounds with an open mind, when the team members are encouraged to speak one's mind freely, his psychological security will be higher. Mutual trust means R \& D personnel has a positive expected of relationship between each other, willing to bear the risks by maintaining the relationship which can produce organizational citizenship behaviors and enhance the cohesion of active collaboration. Knowledge sharing means that team members need to get rid of consciousness to ensure that the key of tacit knowledge within the organization share and accumulate effectively. Team learning should have the characteristic of learning with dialogue and take dialogue and mutual learning process into spiral cycle.

In the process of exchange of information and knowledge, it also need reduce perceived risk of interpersonal relationship from the institution and technology. First, establishment incentive mechanism of the opinions and suggestions, give advice provider corresponding reward. Second , improve the hardware facilities, such as the establishment of organization network, make full use of the network characteristics of quickness and anonymity to rich R \& D team internal communication channels. Three, construct open knowledge base and collect staff's knowledge within the team. Four, find information "brokers" to convey the opinions and suggestions. Five, improve management R \& D personnel time out of the work, such as the association and details layout of the restaurant to provide opportunities for the team.

\section{The Implementation of Humane Management to Reduce $R$ \& D Personnel Perception of Organizational Politics}

The working characteristics of $\mathrm{R} \& \mathrm{D}$ determines the organization shall take the humane management mode, humane management should base on organization trust and shared vision, pay attention to individuals' needs and emotions, and make team members consider the opinions of the others through communication and interaction of emotion. Humane management requires managers show the intention and ability to trust, which is conducive to the rapid start-up and development of subordinates' trust and reduction of closed tendency, so as to show more organizational citizenship behavior. To the scientific research personnel, one of the important connotations of the humane management is to ensure fair in the organization to reduce the members of the perception of organizational politics. The perception of organizational politics refers to a variety of organization members use power or resources to get its own interest including multiple dimensions of political action, clique, supervisors' unfair behavior and promotion policies which will lead to mental resources to suspicion and internal friction and inhibit the role of improving the performance of individual. Members of the perception of organizational politics are closely related to environmental and organizational factors. Research shows that, if the members of the organization participate in decision-making and with higher work autonomy, feedback and cooperation, his perception of organizational politics is low; and higher centralization in organization can lead to strong perception of organizational politics. Many enterprises can solicit opinions in technical decisions, but in the management and decision-making, especially to the staff incentive and promotion, they often lack sufficient transparency and then cause members' suspicion and doubt. Therefore, enterprises should try to speak frankly and sincerely in management decisions, establish the mechanism for organization members to participate in the decision making system. In the work design, they should pay attention to work autonomy while highlighting the overall objectives of the organization and establishment of internal 
feedback channels to encourage members to speak his mind freely. In the salary structure, they should avoid the competitive salary and incentive members take knowledge sharing and cooperative behavior to cultivate the atmosphere of trust in the organization in daily communication. In the feedback mechanism, they should ensure that the communication of members' opinions and suggestions, so that the information provider can see the effect generated by the information, and protect and enhance the enthusiasm of the members. In the training design, they should develop new projects in the training base to form team cooperation and innovation consciousness.

\section{Control Of Organizational Learning Competition}

Organizational learning competition can effectively accumulate knowledge, but also can strengthen the competitive behavior within the organization and lead to close tendency. Therefore, enterprises need to make adjustments in the goal setting to avoid competition lead to negative results. For example, set different goals by individual learning ability, ensure the rationality of team norms, support exploratory point of view, take competition between the colleagues to between organizations.

\section{Take Psychological Health Education to Reduce the Negative Inside Energy of R \& D Personnel}

Psychology research shows, the individual performance is determined not only by the knowledge, but also psychological factors etc.. Relatively speaking, R \& D personnel with higher education are more easily by the external difficulties caused by psychological frustration. Therefore, it need to introduce mental health education into organization to deal with the negative energy in $\mathrm{R} \& \mathrm{D}$ personnel. Foreign employee assistance program (EAP) provides a useful reference. Employee assistance program is a systematic long-term welfare and support designed for the employees in the enterprises to improve the working mood, strengthen confidence, deal with interpersonal relationship properly.

\section{Increase R \& D Personnel Psychological Security By Inter Organizational Cooperation}

The development of high technology enterprise needs an open mind and cooperation between organizations, especially for the complex product system innovation, knowledge joint is becoming the norm. The common cross organizational innovations has two forms IUR cooperative innovation and organization innovation "projects". Inter Organizational Innovation on the one hand need the multi subject participation, the main difference between different knowledge allows developers get more mature opinion easily, on the other hand, interactive game between organization and R \& D personnel is relatively short, $\mathrm{R} \& \mathrm{D}$ personnel has little concerns of interpersonal relationship risk and express their true thoughts about the problem.

In short, the construction of $\mathrm{R} \& \mathrm{D}$ team psychological safety is a systematic project involving all elements of the organization, such as managers, team and external intelligence, and so on. Only in this way, we can get rid of the R \& D team internal psychological barriers to maximize the value of intellectual resources.

\section{Acknowledgement}

This research was financially supported by Science and Technology Project of Weifang tobacco Institute(Research on the appraisal and incentive mechanism optimization of customer manager under the guidance of promotion)

\section{References}

[1] Edmondson, Psychological safety and learning behavior in work teams. Administrative Science Quarterly, 44(1999) 350-383.

[2] Peng \& Ling,Relationship between team psychological safety and learning of dialogue[J]. The vitality of enterprises, 9 (2007) 48-49.

[3] X. P.Ren, Occupation burnout and management countermeasures of scientific research personnel[J]. China technology forum, 12 (2008)120-123. 